\title{
$K$-GROUPS GENERATED BY $K$-SPACES
}

\author{
BY
}

\author{
ERIC C. NUMMELA
}

\begin{abstract}
A $K$-group $G$ with identity $e$ is said to be generated by the $K$-space $X$ if $X$ is a subspace of $G$ containing $e, X$ algebraically generates $G$, and the canonical morphism from the Graev free $K$-group over $(X, e)$ onto $G$ is a quotient morphism. An internal characterization of the topology of such a group $G$ is obtained, as well as a sufficient condition that a subgroup $H$ of $G$ be generated by a subspace $Y$ of $H$. Several illuminating examples are provided.
\end{abstract}

0 . Introduction. In recent years the category $K$ of compactly generated spaces has received attention as being a very convenient setting for investigating various algebraic-topological notions [7], [10], [11]. It is equally as good a setting for topological-algebraic investigations, since such objects as free $K$-modules and tensor products of $K$-modules can be constructed in the category $K$. Indeed, a large part of classical homological algebra can be established in this setting, relative to epimorphisms which admit sections [9]. This article contains an abbreviated account of the construction of free $K$-groups (see [4] for a complete construction), together with a more extensive investigation of a generalization of free $K$-groups. More specifically, this investigation concerns the notion of a $K$-group which has the finest $K$-group topology compatible with a given subspace $X$ which algebraically generates $G$. Although the finest $K$-group topology is initially obtained via a quotient morphism with domain a free $K$-group, an internal characterization is later provided, as well as a sufficient condition that a subgroup $H$ of $G$ have the finest $K$-group topology compatible with a subspace $Y$ of $H$. In the case that $G$ is free over $X$ and $Y$ is a $k_{\omega}$-space, this last result provides a generalization of theorems by Mack, Morris and Ordman [5, Theorem 3] and Graev [3, Theorem 10].

1. Preliminaries. Let $T$ denote the category of all topological spaces and continuous functions. A continuous function $\tau: C \rightarrow X$ with $C$ compact (which means $T_{2}$ ) is called a test function for $X$. Following McCord [7], we say a subset $A$ of a space $X$ is compactly closed if $\tau^{-1}(A)$ is closed in $C$ for every test function $\tau: C \rightarrow X$; a $k$-space is a space in which every compactly closed

Received by the editors March 9, 1973 and, in revised form, January 22, 1974.

AMS (MOS) subject classifications (1970). Primary 20E05, $22 \mathrm{A05}$.

$K e y$ words and phrases. $K$-space, $K$-group, free group. 
subset is closed; a space $X$ satisfies the weak Hausdorff $\left(t_{2}\right)$ property if $\tau(C)$ is closed (and hence compact) in $X$ for every test function $\tau$; a $t_{2} k$-space is compactly generated. (Note that $T_{2}$ implies $t_{2}$, and $t_{2}$ implies $T_{1}$, with both implications proper.) We call compactly generated spaces $K$-spaces, and denote by $K$ the full subcategory of $T$ determined by the $K$-spaces. Note that a $K$ space is the colimit (in $T$ or $K$ ) of its compact subsets ordered by set inclusion. (We shall always assume that our colimits are taken over directed indexing sets.)

By declaring all compactly closed subsets to be closed, we obtain a functor $k$ from topological spaces to $k$-spaces which is adjoint to the forgetful functor, and hence preserves products. That is, the categorical product of $k$-spaces $X$ and $Y$ is $X \times Y=k\left(X \times{ }_{t} Y\right)$, where $\times_{t}$ denotes the product in $T$. Since any topology finer than a $t_{2}$ topology is also $t_{2}$, this also defines the product in $K$. There are many circumstances under which $X \times Y=X \times{ }_{t} Y$; e.g., if $X$ is locally compact, or if both $X$ and $Y$ are first countable.

We denote by $K_{0}$ the category of pointed $K$-spaces. (We do not insist that our base-points be neighborhood deformation retracts, as in $\S 3$ of [7].)

A $K$-group is a group in $K$; i.e., $X$ is a $K$-group if it is a $K$-space and an algebraic group such that the function $(x, y) \rightarrow x y^{-1}: X \times X \rightarrow X$ is continuous. A topological group need not be a $K$-group, although if $X$ is a $t_{2}$ topological group, then $k X$ is a $K$-group. Also, a $K$-group need not be a topological group, although locally compact and first countable $K$-groups are topological groups. (In fact, we shall see in $\$ 2$ that a $K$-group need not be uniformizable. See Example 1.) Let (A) $G$ denote the category of all (abelian) $K$ groups and continuous group homomorphisms.

A $k_{\omega}$-space is a space which is the colimit (in the category $T$ ) of an expanding sequence of compact spaces. Since a compact space is normal, the following lemma shows that a $k_{\omega}$-space is $T_{2}$.

LEMMA 1.1. Let $X$ be a space which is the colimit (in $T$ ) of an expanding sequence $\left(X_{n}\right)_{n=0}^{\infty}$ of closed normal subspaces. Then $X$ is normal.

Proof. We may use the proof of Theorem 5 of [3].

Now, by Lemma 9.2 of [10], we see that a $k_{\omega}$-space is a $T_{2} K$-space. (Thus $k_{\omega}$-spaces may be regarded as colimits in either $T$ or $K$ of expanding sequences of compact spaces.) Moreover, the product in $K$ of two $k_{\omega}$-spaces is the usual topological product. (This can be proved via an easy adaptation of the proof of Lemma 2.1 of [8], or see [12].) Thus a $k_{\omega}$-group is a topological group.

Question 1. Which $K$-groups are topological groups; i.e., what is the intersection of the category of $K$-groups and the category of $T_{2}$ topological groups? 
2. Free $K$-groups. We take as known the concepts of free topological group due to A. A. Markov [6] and M. I. Graev [3]. By way of notation, FX (ZX) will denote the Markov free (free abelian) topological group over the completely regular space $X$, and $F\left(X, x_{0}\right)\left(Z\left(X, x_{0}\right)\right)$ will denote the Graev free (free abelian) topological group over the pointed completely regular space $\left(X, x_{0}\right)$. Since Graev free topological groups are independent of base-point, we may speak of the Graev free topological group over $X$. If $X$ is a $k_{\omega}$-space, then so are $F X, Z X$, $F\left(X, x_{0}\right)$, and $Z\left(X, x_{0}\right)$, for any $x_{0} \in X$. (See [5]; the abelian case is also proved in [12].)

The category of $K$-groups bears many similarities to the category of topological groups, but results about $K$-group topologies must often be obtained in a different manner, since the standard uniform structure of a topological group is not available. We first indicate the construction of free $K$-groups, and then use free $K$-groups to obtain finest $K$-group topologies. This is in complete contrast to the situation for topological groups, where the most economical way to construct free objects is to take the supremum of a set of group topologies on an algebraic free group.

Categorically speaking, free objects are determined via a coadjoint (i.e., left adjoint) to a forgetful functor. In the case at hand, we have forgetful functors from the category $G(A)$ to $K$ and $K_{0}$, where the identity element of a $K$ group is always taken as base-point. The corresponding free objects are called Markov and Graev free (free abelian) $K$-groups, respectively, in honor of their topological counterparts. A specific construction of free $K$-groups, inspired by the work of McCord [7, §6], proceeds as follows. (See [4] for a slightly different, but much more detailed, approach.)

Let $\left(X, x_{0}\right)$ be a pointed $K$-space, and let $F\left(X, x_{0}\right)$ be the algebraic free group over the set underlying $X-\left\{x_{0}\right\}$, identifying $x_{0}$ with $e$, the identity of $F\left(X, x_{0}\right)$. We let $F_{n} X$ denote the set of words in $F\left(X, x_{0}\right)$ of reduced length less than or equal to $n$, and topologize $F_{n} X$ as a quotient of the $n$-fold product of $X \times\{-1,1\}$. Then each $F_{n} X(0 \leqslant n<\infty)$ is a $K$-space and is closed in $F_{n+1} X$. We give $F\left(X, x_{0}\right)$ the colimit topology (in $\left.T\right)$. Then $F\left(X, x_{0}\right)$ is a $K$-group, and the canonical inclusion $\eta_{X}:\left(X, x_{0}\right) \rightarrow F\left(X, x_{0}\right)$ is an embedding onto a closed subspace. It is easy to verify that $F\left(X, x_{0}\right)$ satisfies the required universal property, and is hence the Graev free $K$-group over $\left(X, x_{0}\right)$ (or simply over $X$, if the base-point is unimportant).

Since $K$-spaces may be $T_{2}$ and not completely regular, $K$-groups need not be completely regular, and hence not topological groups.

EXAMPLE 1. Let $X$ be the set of real numbers with the topology generated by the usual open intervals together with the set $Q$ of rational numbers. Then $X$ is $T_{2}$, but not regular, since the closed set $I=X-Q$ cannot be separated 
from the point 1, say. (See [2, Example 2, p. 141].) Since $X$ is first countable, it is a $T_{2} K$-space. Hence the Graev free $K$-group over $X$ exists and is not a topological group.

We note further that $Y=X / I$ is a $K$-space [7, Proposition 2.5, p. 277] which is not $T_{2}$, by the preceding paragraph. Hence the Graev free $K$-group over $Y$ is a $K$-group which is not $T_{2}$ (although, of course, it is $t_{2}$ and hence $\left.T_{1}\right)$.

The following question, suggested by the preceding example, is related to Question 1.

Question 2. Must uniformizable $K$-groups be topological groups?

We may now obtain $Z\left(X, x_{0}\right)$, the Graev free abelian $K$-group over $\left(X, x_{0}\right)$, either by taking $F\left(X, x_{0}\right)$ modulo its commutator subgroup (which can be shown to be closed); or by using the specific construction of $\S 6$ of [7], on which the construction of $F\left(X, x_{0}\right)$ is modeled; or by topologizing $Z_{n} X$ (the set of words in $Z\left(X, x_{0}\right)$ of length less than or equal to $\left.n\right)$ as a quotient of $[X \times\{-1,1\}]^{n} / S_{n}$, where $S_{n}$ is the symmetric group on $n$ letters acting on $[X \times\{-1,1\}]^{n}$ in the canonical way.

It is now easy to see that, given any $K$-space $X$, the Markov free $K$-group $F X$ over $X$ exists. Indeed, if $\{*\}$ is a singleton $K$-space with $* \notin X$, then $F X \cong F(X \cup\{*\}, *)$. An analogous statement holds for the abelian case.

We conclude this section by noting that a free $K$-group is the colimit (in $T$ or $K$ or $G$ !) of free $k_{\omega}$-groups. More specifically, let $\left(X, x_{0}\right)$ be a pointed $K$ space, and let $C$ be any family of compact subsets of $X$ such that $X=$ colim $C$. (This colimit may be taken in $T$ or $K$; however, recall our convention that $C$ must be directed.) We assume, without loss of generality, that $x_{0} \in C$ for every $C \in C$. Since coadjoints preserve colimits, then $F\left(X, x_{0}\right)$ is isomorphic to the colimit in $G$ of the family $\left\{F\left(C, x_{0}\right)\right\}$, where the morphisms in the colimit diagram are induced by the inclusions of one compact set into another. The following theorem provides an even more specific view of this isomorphism.

THEOREM 2.1. In the context of the above paragraph, let $\langle C\rangle$ denote the subgroup of $F\left(X, x_{0}\right)$ algebraically generated by $C \in C$. Let $\phi_{C}: F\left(C, x_{0}\right) \rightarrow$ $F\left(X, x_{0}\right)$ be the (unique) morphism in $G$ induced by the inclusion $C \rightarrow X$. Then each $\langle C\rangle$ is closed in $F\left(X, x_{0}\right)$, and hence is a $K$-group. Furthermore, for any subset $A$ of $F\left(X, x_{0}\right)$, the following conditions are equivalent.

(1) $A$ is closed.

(2) $A \cap\langle C\rangle$ is closed for all $C \in \mathrm{C}$.

(3) $A \cap \phi_{C}\left(F_{n} C\right)$ is closed for all $C \in C, n \in N=\{0,1,2, \cdots\}$.

This shows that for each $C \in C,\langle C\rangle \cong F\left(C, x_{0}\right)$; in particular, each $\langle C\rangle$ is a $k_{\omega^{-}}$ group. Moreover, $F\left(X, x_{0}\right)=\operatorname{colim}\langle C\rangle$, relative to the inclusion morphisms (in $T$, $K$, or G). 
Proof. Let $C \in C$. To show that $\langle C\rangle$ is closed, we must show that $\langle C\rangle \cap$ $F_{n} X=\phi_{C}\left(F_{n} C\right)$ is closed for all $n \in N$. But $F_{n} C$ is compact. Thus $\phi_{C}\left(F_{n} C\right)$ is compact, thus closed, and hence $\langle C\rangle$ is closed.

Now it is clear that (1) implies (2) and (2) implies (3).

We prove (3) implies (1). Assume $A \cap \phi_{C}\left(F_{n} C\right)$ is closed for all $C \in C$, $n \in N$. We must show $A \cap F_{n} X$ is closed for all $n$. It suffices to show that $B_{n}=\mu_{n}^{-1}\left(A \cap F_{n} X\right)$ is closed in $(X \times\{-1,1\})^{n}$, where $\mu_{n}:(X \times\{-1,1\})^{n} \rightarrow$ $F_{n} X$ is the quotient morphism. But since $(X \times\{-1,1\})^{n}$ is a $K$-space, $B_{n}$ will be closed if it intersects every compact subset in a closed set. It is clearly enough to look at "rectangular" compact subsets, and we may as well assume the "sides" of the rectangle are all the same, say $C \times\{-1,1\}$ for some $C \in \mathcal{C}$ (since $C$ is directed). Now

$$
B_{n} \cap(C \times\{-1,1\})^{n}=\mu_{n}^{-1}\left(A \cap F_{n} X \cap \phi_{C}\left(F_{n} C\right)\right)=\mu_{n}^{-1}\left(A \cap \phi_{C}\left(F_{n} C\right)\right),
$$

which is closed.

The remainder of the theorem follows at once.

We remark that analagous results may be obtained by replacing $F\left(X, x_{0}\right)$ by $F X, Z\left(X, x_{0}\right)$, or $Z X$.

3. $K$-groups generated by $K$-spaces. With free $K$-groups now at our disposal, we can obtain "finest" $K$-group topologies. However, we first establish some notation important in the remainder of this section. We will always use $G$ to denote a $K$-group with topology $\tau$. For any subset $X$ of $G$, let $\langle X\rangle$ denote the subgroup of $G$ (with the subspace topology) algebraically generated by $X$. If $X$ is a $K$-space and contains $e$, we denote by $\phi=\phi_{X}: F(X, e) \rightarrow G$ the (unique) morphism in $G$ induced by the inclusion $X \rightarrow G$. (The condition that $e \in X$ is not necessary, since we could always adjoin $e$ to $X$; however, it is more convenient to work with Graev free $K$-groups than Markov free $K$-groups.) We set $X_{n}=\phi\left(F_{n} X\right)$ for all $n \in N$. Note that $\langle X\rangle=G$ if and only if $\phi$ is surjective. If $\langle X\rangle=G$, we denote by $\tau^{\prime}$ the topology on $G$ induced by declaring $\phi$ to be a quotient morphism. Then $\tau \subset \tau^{\prime}$ and $\tau^{\prime}|X=\tau| X$.

We assume now that $X$ is a $K$-space, that $e \in X$, and that $\langle X\rangle=G$.

LEMma 3.1. The topology $\tau^{\prime}$ is a $K$-group topology on $G$.

Proof. The topology $\tau^{\prime}$ is a $k$-topology, since $\phi$ is a quotient morphism; it is $t_{2}$, since $\tau \subset \tau^{\prime}$ and $\tau$ is $t_{2}$; and it is a $K$-group topology, since the product of two quotient morphisms (in $K$ ) is again a quotient morphism.

THEOREM 3.2. The topology $\tau^{\prime}$ is the finest $K$-group topology on $G$ compatible with the topology of $X$. 
Proof. If $\tau^{\prime \prime}$ is a $K$-group topology on $G$ with $\tau^{\prime \prime}|X=\tau| X$, then the inclusion $j: X \rightarrow\left(G, \tau^{\prime \prime}\right)$ is continuous. Hence $j$ extends to a (unique) morphism $\bar{j}: F(X, e) \rightarrow\left(G, \tau^{\prime \prime}\right)$ in $G$. But $\operatorname{Ker}(\bar{j})=\operatorname{Ker}(\phi)$. Hence there exists a unique morphism $\bar{\phi}:\left(G, \tau^{\prime}\right) \rightarrow\left(G, \tau^{\prime \prime}\right)$ in $G$ such that $\bar{\phi} \phi=\bar{j}$. That is, $\tau^{\prime \prime} \subset \tau^{\prime}$.

EXAMPLE 2 . It can be the case that $\tau^{\prime}$ is strictly finer than $\tau$. For example, let $G$ denote the multiplicative group of positive rational numbers, with $\tau$ the usual topology. Then $G$ is first countable, and hence a $K$-group. Let $X=$ $\{p \mid p$ is a prime $\cup\{1\}$. Then $\langle X\rangle=G$; and $X$ is discrete, hence a $K$-space. Thus $F(X, 1)$ is discrete, and hence the topology $\tau^{\prime}$ induced on $G$ by $\phi$ is also discrete. But $\tau$ is not discrete, and thus $\tau^{\prime}$ is strictly finer than $\tau$.

It seems appropriate to single out those $K$-groups $G$ for which $\tau=\tau^{\prime}$. Thus we say a $K$-group $G$ is generated by the $K$-space $X$ if

(1) $X$ is (homeomorphic to) a subspace of $G$ containing $e$,

(2) $\langle X\rangle=G$, and

(3) the inclusion $X \rightarrow G$ induces a quotient morphism $F(X, e) \rightarrow G$. As shown by Example 2, conditions (1) and (2) may be satisfied without condition (3). We note the two natural extremes illustrated by

(a) $F\left(X, x_{0}\right)$ is generated by $X$ for any pointed $K$-space $\left(X, x_{0}\right)$, and

(b) $G$ is generated by $G$ for any $K$-group $G$.

Recalling the original definition of the topology on a Graev free $K$-group, we might ask if there is not a similar "internal" characterization of the topology on a $K$-group $G$ generated by the $K$-space $X$. An affirmative answer is obtained by carrying down the structure from $F(X, e)$; i.e., by replacing $F_{n} X$ by $X_{n}=$ $\phi\left(F_{n} X\right)$. The expected mapping property is obtained, as well.

THEOREM 3.3. Let $G$ be a $K$-group with topology $\tau$, and let $X$ be a subspace of $G$ such that $X$ is a $K$-space, $e \in X$, and $\langle X\rangle=G$. Then the following conditions are equivalent.

(1) $G$ is generated by $X$.

(2) $G=\operatorname{colim} X_{n}$ (in $T$ or K).

(3) If $H$ is a $K$-group and if $f: G \rightarrow H$ is an algebraic homomorphism, then $f$ is continuous if and only if $f \mid X$ is continuous.

Proof. (1) $\Rightarrow$ (2): It is obvious that if $A \subset G$ is closed, then $A \cap X_{n}$ is closed in $X_{n}$ for each $n \in N$. Conversely, let $A \subset G$ and suppose $A \cap X_{n}$ is closed in $X_{n}$ for each $n$. Then $\phi^{-1}\left(A \cap X_{n}\right)=\phi^{-1}(A) \cap \phi^{-1}\left(X_{n}\right)$ is closed in $\phi^{-1}\left(X_{n}\right)$ for each $n$. Since $F_{n} X$ is closed and $F_{n} X \subset \phi^{-1}\left(X_{n}\right)$, then $\phi^{-1}(A) \cap F_{n} X$ is closed for each $n$. Hence $\phi^{-1}(A)$ is closed in $F(X, e)$. Since $\phi$ is a quotient morphism, then $A$ is closed.

(2) $\Rightarrow$ (3): Let $H$ be a $K$-group and $f: G \rightarrow H$ an algebraic homomor- 
phism. Certainly, if $f$ is continuous, then $f \mid X$ is continuous. Conversely, suppose $f \mid X$ is continuous. It suffices to show that $f \mid X_{n}$ is continuous for each $n$. For $z \in X^{-1}$, we have $f(z)=f\left(z^{-1}\right)^{-1}$. Since $z^{-1} \in X$ and inversion is continuous in $H, f \mid X^{-1}$ is also continuous. Thus $f \mid X_{1}$ is continuous. Now for $z \in X_{n}$, we may write $z=x_{1} x_{2} \cdots x_{n}$, with $x_{1}, x_{2}, \cdots, x_{n} \in X_{1}$. Then $f(z)=f\left(x_{1}\right) f\left(x_{2}\right) \cdots f\left(x_{n}\right)$. Since multiplication in $H$ is continuous, $f \mid X_{n}$ is continuous.

(3) $\Rightarrow$ (1): Let $\tau^{\prime}$ be the topology on $G$ induced by $\phi$. Then $\tau \subset \tau^{\prime}$ and $\tau^{\prime}|X=\tau| X$. Hence the identity homomorphism from $(G, \tau)$ to $\left(G, \tau^{\prime}\right)$ is continuous. But this means $\tau^{\prime} \subset \tau$. Hence $\tau=\tau^{\prime}$, and thus $G$ is generated by $X$.

Remark 3.4. Note that $X$, and hence $X_{n}$ for any $n \in N$, need not be closed. For example, let $G$ be the additive group of real numbers with the usual topology, and let $X=\left[0,1\left[\right.\right.$. Then $\left.X_{n}=\right]-n, n[$ for $n>0$. Condition (2) of Theorem 3.3 is clearly satisfied. (This example, with $X=10,1$, appears in the first remark of [5].)

REMARK 3.5. The situation dealt with in the above theorem is slightly more involved than the construction of Graev free $K$-groups. For let $G$ be an arbitrary algebraic group, let $X$ be a subset of $G$ with $e \in X$ and $\langle X\rangle=G$, and suppose $X$ is endowed with a $K$-topology $\tau_{X}$. Condition (2) of Theorem 3.3 provides an internal way of constructing the finest $K$-group topology on $G$ compatible with $\tau_{X}$, provided that there is at least one $K$-group topology $\tau$ on $G$ compatible with $\tau_{X}$. In the absence of this proviso, the topology $\tau^{\prime}$ obtained on $G$ as a quotient of $F(X, e)$ (i.e., by imitating the construction at the beginning of $\S 2$ ) will be a $k$-group topology, but not necessarily a $K$-group topology; moreover, $\tau^{\prime} \mid X$ may be strictly coarser than $\tau_{X}$.

ExAmple 3. Let $Z$ denote the additive group of integers. Let $X$ be the nonnegative integers with the following topology: give the positive integers the discrete topology, and adjoin 0 as a point at infinity. Then $X$ is compact, hence a $K$-space. Take $G=Z$.

For ease of notation, we will topologize $G$ via the quotient morphism $\psi: Z(X, 0) \rightarrow G$. (Recall that $Z(X, 0)$ is a quotient of $F(X, 0)$.) Note that $Z(X, 0)$ is a $K$-group and a topological group (since $X$ is compact), and thus $G$ is a $k$-group and a topological group. Moreover, $G$ is quasi-compact, since $\psi\left(X_{1}\right)=G$, and $X_{1}$ is compact. But $G$ is not $T_{2}$, since by Corollary 2 of [1], the only locally compact group topology on $Z$ is the discrete topology.

To determine the topology on $G$, we must be more explicit regarding $Z(X, 0)$. We write words in $Z(X, 0)$ as finite formal sums $\epsilon_{1} a_{1} \oplus \cdots \oplus \epsilon_{n} a_{n}$, where $a_{1}, \cdots, a_{n} \in X$ and $\epsilon_{i}= \pm 1(1 \leqslant i \leqslant n)$. Thus $\psi\left(\epsilon_{1} a_{1} \oplus \cdots \oplus \epsilon_{n} a_{n}\right)=$ $\epsilon_{1} a_{1}+\cdots+\epsilon_{n} a_{n} \in G$. For $p \in G$, with $p>0$, consider the sequence 
$\bar{p}=((n+p) \oplus(-n))_{n=0}^{\infty}$. The sequence $(n)$ converges to 0 in $X$, and hence $(n+p)$ and $(-n)$ converge to 0 . Thus, by the continuity of addition in $Z(X, 0)$, $\bar{p}$ converges to 0 . But $\psi(\vec{p})$ is the constant sequence $(p)$. Since $\psi$ is continuous, $(p)$ converges to 0 in $G$. A similar argument shows that for $p \in G$ with $p<0$, the constant sequence $(p)$ converges to 0 in $G$. Hence the closure of $\{0\}$ in $G$ is all of $G$. That is, $G$ is indiscrete.

REMARK 3.6. As a continuation of the above remark, we compare our notion of "generated by" with Markov's "primary strict realization" of a completely regular space $[6, \S 7]$. Translating Markov's notion into the category $K_{0}$, we have the following: Let $\left(X, x_{0}\right)$ be a pointed $K$-space, let $R$ be an equivalence relation on $X$, let $G$ be a $K$-group, and let $\theta: X \rightarrow G$ be a morphism in $K_{0}$ (i.e., $\left.\theta\left(x_{0}\right)=e\right)$. The pair $(\theta, G)$ is a strict realization of $(X, R)$ if

(1) $\langle\theta(X)\rangle=G$, and

(2) $(x, y) \in R$ implies $\theta(x)=\theta(y)$.

The strict realization $(\theta, G)$ is primary if

(3) it is universal with respect to strict realizations; i.e., if $(\psi, H)$ is a strict realization of $(X, R)$, then there exists a unique morphism $\bar{\psi}: G \rightarrow H$ in $G$ such that $\bar{\psi} \theta=\psi$.

A primary strict realization of $(X, R)$ is obtained by taking $F(X, e)$ modulo the smallest closed congruence containing the canonical image of $R$. (This is essentially Lemma 50 of [6].)

Suppose $G$ is a $K$-group, $X$ is a subspace of $G$ which is a $K$-space, $e \in X$, $\langle X\rangle=G$, and $R$ is the restriction to $X \times X$ of the kernel relation of $G$. Then $(i, G)$, where $i: X \rightarrow G$ is inclusion, is a primary strict realization of $(X, R)$ if and only if $G$ is generated by $X$.

If however, we take $X$ as in Example 3, with $R$ the restriction to $X \times X$ of the kernel relation induced by addition, then the primary strict realization of $(X, R)$ is a one element group.

The following nice property of a $K$-group $G$ generated by the $K$-space $X$ may be easily proved by adapting the proof of Lemma 9.3 of [10].

Proposition 3.7. If $G$ is a $K$-group generated by the $K$-space $X$, and if $C$ is a compact subset of $G$, then there exists $n \in N$ such that $C \subset X_{n}$.

By Theorem 2.1 we know that a Graev free $K$-group is the colimit of subgroups which are $k_{\omega}$-groups. In general, we can not expect such nice behavior, as Example 4 below will show. However, we do have the following result.

Proposition 3.8. Let $G$ be a $K$-group generated by the $K$-space $X$, let $C$ be a family of compact subsets of $X$ containing $e$ such that $X=$ colim C, and suppose each $\langle C\rangle$ is a $K$-group. (This is the case, for example, if each $\langle C\rangle$ 
is closed in $G$.) Then $G=\operatorname{colim}\langle C\rangle$, relative to the inclusion morphisms. (This colimit may be taken in $T, K$, or $G$.)

Proof. Simply use the canonical morphisms $F(C, e) \rightarrow\langle C\rangle$ and $F(X, e) \rightarrow$ $G$, together with the fact that $F(X, e)=\operatorname{colim} F(C, e)$.

EXAMPLE 4. In the context of Proposition 3.8, it is in general false that each $\langle C\rangle$ must be a $k_{\omega}$-group. For consider again Example 2. The $K$-group $G$ is generated by the $K$-space $G$. Now let $C$ be any compact subset of $G$ containing 1 . If $\langle C\rangle$ is a $k_{\omega}$-group, then it is locally compact, since a first countable $k_{\omega}$-space is locally compact. But $G$ is algebraically a free abelian group; hence $\langle C\rangle$ is also. Thus by Dudley's result [1, Corollary 2], $\langle C\rangle$ is discrete. Now if $G$ were the colimit of a family of $\langle C\rangle$ 's (relative to the inclusion morphisms) as it would be by Proposition 3.8 if each $\langle C\rangle$ were a $k_{\omega}$-group, then $G$ would be discrete, which is false.

Indeed, this shows that $G$ is not a colimit of any family of subgroups which are $k_{\omega}$-groups. [This example is a modification of an example due to E. T. Ordman communicated to the author by the referee.]

Question 3. Which $K$-groups are colimits of some family of subgroups (relative to inclusion morphisms) which are $k_{\omega}$-groups? Example 4 shows that not all $K$-groups are. What about locally compact groups?

If $G$ is generated by $X$, we might ask when a subspace $Y$ of $G$ generates $\langle Y\rangle$. The following theorem gives some guidelines.

THEOREM 3.9. Let $G$ be a $K$-group generated by the $K$-space $X$. Let $Y$ be a subspace of $G$ such that $Y$ is a $K$-space, $e \in Y$, and $Y_{m}$ is closed in $G$ for all $m \in N$. If for each $n \in N$ there exists $m \in N$ such that $\langle Y\rangle \cap X_{n} \subset$ $Y_{m}$, then $Y$ generates $\langle Y\rangle$ and $\langle Y\rangle$ is closed in $G$.

Proof. Suppose $A \subset\langle Y\rangle$ and $A \cap Y_{m}$ is closed for all $m \in N$. Let $n \in N$, and let $m \in N$ be such that $\langle Y\rangle \cap X_{n} \subset Y_{m}$. Then

$$
A \cap X_{n}=A \cap\langle Y\rangle \cap X_{n}=A \cap\langle Y\rangle \cap X_{n} \cap Y_{m}=A \cap Y_{m} \cap X_{n} .
$$

Since $A \cap Y_{m}$ is closed, $A \cap X_{n}$ is closed in $X_{n}$. Hence $A$ is closed in $G$, and also in $\langle Y\rangle$.

Taking $A=\langle Y\rangle$, we see that $\langle Y\rangle$ is closed. Taking $A$ to be arbitrary, we see that $Y$ generates $\langle Y\rangle$.

In practice, the criterion that each $Y_{m}$ should be closed may be difficult to verify. However, it is automatically satisfied if $Y$ is compact. Nevertheless, the following example shows that even if $Y$ is a compact subset of $X, Y$ may fail to generate $\langle Y\rangle$ and $\langle Y\rangle$ may fail to be closed.

EXAMPLE 5. Let $G$ be the additive group of real numbers with the usual 
topology, and let $X=[0,1[$. As noted earlier, then $G$ is generated by $X$, with $\left.X_{n}=\right]-n, n[$ for each $n>0$. Let $Y=\{1 / p \mid p$ is a prime $\cup\{0\}$. Then $Y$ is a compact subset of $X$, and $Y_{m}$ is compact, hence closed in $G$, for all $m \in N$. However, since the sum $\Sigma 1 / p$ diverges, every real number is in the closure of $\langle Y\rangle$. Thus $\langle Y\rangle$ is dense in $G$, and, of course, not closed. Another consequence of the density is that $\langle Y\rangle \cap\} n, n$ [ cannot be contained in $Y_{m}$ for any $m$. We also see that $\langle Y\rangle$ is not generated by $Y$; for if it were, then it would be a $k_{\omega}$-group, hence locally compact (since it is first countable), hence closed.

4. Free $K$-groups revisited. In case $G=F(X, e)$, we use Theorem 2.1 to obtain a generalization of Theorem 3 of [5].

THEOREM 4.1. Let $G$ be a $K$-group generated by the $K$-space $X$, and suppose $G=F(X, e)$. Let $C$ be any family of compact subsets of $X$ containing $e$ such that $X=\operatorname{colim} C$. Let $Y$ be a subspace of $G$ containing $e$ which is a $K$ space, and let $D$ be any family of compact subsets of $Y$ containing $e$ such that $Y=$ colim D. Consider the following conditions:

(1) $\langle Y\rangle$ is generated by $Y$ and $\langle Y\rangle$ is closed in $G$;

(2) for each $C \in C$ and $n \in N$, there exist $D \in D$ and $m \in N$ such that $\langle Y\rangle \cap C_{n} \subset D_{m}$.

Then (2) implies (1). In particular, if (2) holds and $\langle Y\rangle$ is algebraically free over $Y-\{e\}(e . g .$, if $Y \subset X)$, then $\langle Y\rangle=F(Y, e)$, and thus $Y$ is closed in $G$. Conversely, if the family $D$ is countable, then (1) implies (2).

Proof. We first prove that (2) implies (1). Suppose $A \subset\langle Y\rangle$ and $A \cap$ $Y_{m}$ is closed in $Y_{m}$ for all $m \in N$. We wish to show that $A$ is closed in $G$. By condition (3) of Theorem 2.1, it is sufficient to show that $A \cap C_{n}$ is closed for all $C \in C, n \in N$. (Recall that $C_{n}=\phi_{C}\left(F_{n} C\right)$, where $\phi_{C}: F(C, e) \rightarrow F(X, e)$ is induced by the inclusion $C \rightarrow X$.) Let $C \in C$ and $n \in N$ be fixed, and let $D \in D$ and $m \in N$ be such that $\langle Y\rangle \cap C_{n} \subset D_{m}$. Then

$$
A \cap C_{n}=A \cap\langle Y\rangle \cap C_{n}=A \cap\langle Y\rangle \cap C_{n} \cap D_{m}=A \cap D_{m} \cap C_{n} .
$$

Since $A \cap Y_{m}$ is closed in $Y_{m}, A \cap D_{m}$ is closed, and hence $A \cap C_{n}$ is closed. Thus $A$ is closed. Taking $A=\langle Y\rangle$, we see that $\langle Y\rangle$ is closed. Taking $A$ to be arbitrary, we see that $Y$ generates $\langle Y\rangle$.

The "in particular" conclusion of the theorem follows immediately. (If $Y \subset X$, then $\langle Y\rangle \cap C_{n}=\left(Y \cap C_{n}\right.$.)

Suppose now that (1) holds and that $D$ is countable, say $D=\left\{D_{m}\right\}_{m=0}^{\infty}$. We may assume that $D_{0}=\{e\}$, and that the sequence is increasing. Thus $Y$ is a $k_{\omega}$-space. Hence, by the proof of Theorem 2 of [5], the topology of $\langle Y\rangle$ is determined by the family $\left(D_{m}\right)_{m}=\phi_{D_{m}}\left(F_{m}\left(D_{m}\right)\right)$. Now for any $C \in C$ and 
$n \in N,\langle Y\rangle \cap C_{n}$ is a compact subset of $\langle Y\rangle$. Hence $\langle Y\rangle \cap C_{n} \subset\left(D_{m}\right)_{m}$ for some $m \in N$, by Lemma 9.3 of [10]; i.e., (2) holds.

REMARK 4.2. The last assertion of the above theorem provides a converse to Theorem 3 of [5]; together they generalize Theorem 10 of [3] (which is incompletely cited as Corollary 2 of [5]), a theorem about subgroups of free topological groups.

Question 4. In Theorem 4.1, does (1) imply (2) without the condition that $D$ be countable?

\section{REFERENCES}

1. R. M. Dudley, Continuity of homomorphisms, Duke Math. J. 28 (1961), 587-594. MR $25 \# 141$.

2. J. Dugundji, Topology, Allyn and Bacon, Boston, Mass., 1966. MR 33 \#1824.

3. M. I. Graev, Free topological groups, Izv. Akad. Nauk SSSR Ser. Mat. 12 (1948), 279-324; English transl., Amer. Math. Soc. Transl. (1) 8 (1962), 305-364. MR 10, 11.

4. W. F. LaMartin, k-groups, Ph. D. Thesis, Tulane University, New Orleans, La., 1973.

5. J. Mack, S. A. Morris and E. T. Ordman, Free topological groups and the projective dimension of a locally compact group, Proc. Amer. Soc. 40 (1973), 303-308.

6. A. A. Markov, On free topological groups, Izv. Akad. Nauk SSSR Ser. Mat. 9 (1945), 3-64; English transl., Amer. Math. Soc. Transl. (1) 8 (1962), 195-272. MR 7, 7.

7. M. C. McCord, Classifying spaces and infinite symmetric products, Trans. Amer. Math. Soc. 146 (1969), 273-298. MR 40 \#946.

8. J. Milnor, Construction of universal bundles. I, Ann. of Math. (2) 63 (1956), 272284. MR 17, 994.

9. E. C. Nummela, Homological algebra of $K$-modules (submitted).

10. N. E. Steenrod, A convenient category of topological spaces, Michigan Math. J. 14 (1967), 133-152. MR 35 \#970.

11. R. M. Vogt, Convenient categories of topological spaces for homotopy theory, Arch. Math. (Basel) 22 (1971), 545-555. MR 45 \#9323.

12. D. Wigner, Algebraic cohomology of topological groups, Trans. Amer. Math. Soc. 178 (1973), 83-94.

DEPARTMENT OF MATHEMATICS, UNIVERSITY OF FLORIDA, GAINESVILLE, FLORIDA 32611 